\title{
Compact multichannel MEMS based spectrometer for FBG sensing
}

\author{
Ganziy, Denis; Rose, Bjarke; Bang, Ole
}

Published in:

Proceedings of 25th International Conference on Optical Fiber Sensors

Link to article, DOI:

$10.1117 / 12.2264994$

Publication date:

2017

Document Version

Publisher's PDF, also known as Version of record

Link back to DTU Orbit

Citation (APA):

Ganziy, D., Rose, B., \& Bang, O. (2017). Compact multichannel MEMS based spectrometer for FBG sensing. In Proceedings of 25th International Conference on Optical Fiber Sensors (Vol. 10323). [103230B ] SPIE International Society for Optical Engineering. https://doi.org/10.1117/12.2264994

\section{General rights}

Copyright and moral rights for the publications made accessible in the public portal are retained by the authors and/or other copyright owners and it is a condition of accessing publications that users recognise and abide by the legal requirements associated with these rights.

- Users may download and print one copy of any publication from the public portal for the purpose of private study or research.

- You may not further distribute the material or use it for any profit-making activity or commercial gain

- You may freely distribute the URL identifying the publication in the public portal 


\title{
Compact multichannel MEMS based spectrometer for FBG sensing
}

\author{
D. Ganziy*a,, B. Rose ${ }^{\mathrm{a}}$, O. Bang ${ }^{\mathrm{b}}$ \\ ${ }^{a}$ Ibsen Photonics A/S, Ryttermarken 15-21, 3520 Farum, Denmark; \\ ${ }^{b}$ DTU Fotonik, Department of Photonics Engineering, Technical University of Denmark \\ 2800 Kgs. Lyngby, Denmark
}

\begin{abstract}
We propose a novel type of compact multichannel MEMS based spectrometer, where we replace the linear detector with a Digital Micromirror Device (DMD). The DMD is typically cheaper and has better pixel sampling than an InGaAs detector used in the $1550 \mathrm{~nm}$ range, which leads to cost reduction and better performance. Moreover, the DMD is a 2D array, which means that multichannel systems can be implemented without any additional optical components in the spectrometer. This makes the proposed interrogator highly cost-effective. The digital nature of the DMD also provides opportunities for advanced programmable spectroscopy.
\end{abstract}

Keywords: FBG interrogation, MEMS, DMD, DLP, spectroscopy

\section{INTRODUCTION}

Fiber Bragg gratings (FBGs) have become more and more attractive in recent years because they are small, costeffective, easy to manufacture, and insensitive to electromagnetic interference [1]. The basic principle of FBG sensing is to measure the reflected spectrum and to track the FBG peak position. Due to the availability of low-cost telecommunications equipment the most common and commercially available FBGs work in the $1550 \mathrm{~nm}$ range. However, this requires use of expensive $1550 \mathrm{~nm}$ InGaAs array detectors to interrogate the sensors.

Here we propose a novel type of multichannel MEMS based spectrometer, where the linear detector is replaced with a commercial available Digital Micromirror Device (DMD) [2]. The DMD is a 2D mirror array with several hundred thousand microscopic mirrors, that can be set individually in either on or off state to switch selected wavelengths towards a single detector while sending the remaining light to a dump. The DMD is typically cheaper and has better pixel sampling than an InGaAs detector used in the $1550 \mathrm{~nm}$ range, which may lead to cost reduction and better performance. Thanks to the fact that the DMD is a 2D array, multichannel systems can be implemented without any additional optical components, which makes the proposed spectrometer highly cost-effective.

The presence of multiple channels also allows to measure simultaneously several parameters, like temperature, strain, humidity, etc. In addition, the digital nature of the DMD makes it very flexible and provides opportunities for Hadamard spectroscopy, which greatly improves the performance.

\section{OPTICAL DESIGN}

In a standard spectrometer different colors are dispersed by the diffraction gratings across the linear detector. In the DMD the mirrors can be controlled individually, thus the replacement of the detector by the DMD makes it possible to switch out exactly the color required, whereas all other colors are sent to a wavelength dump [3].

The presented spectrometer takes as input a wavelength signal from 1525 to $1570 \mathrm{~nm}$. The optical input contains 4 optical fibers, where each fiber is a standard telecom single mode SM-28 fiber. The selected DMD is the DLP2010NIR produced by Texas Instruments [2] with an $854 \times 480$ array of polarization independent aluminum coated micrometersized mirrors, in an orthogonal layout with $5.4 \mathrm{um}$ mirror pitch. The chip active array size is 4.61 by $2.59 \mathrm{~mm}$. A schematic of the optical layout for the spectrometer is illustrated in Fig. 1 (a). The optical signal enters the device through one of 4 optical fibers mounted in a V-groove assembly. The divergent light from the fibers are collimated by an achromatic lens. Two highly efficient diffraction gratings with the same properties are used to disperse the light. After the gratings the various wavelengths are focused by an achromatic lens onto the DMD surface (see Figure 2 (a)). When the DMD mirror is in the ON state the light is reflected and sent through the same components back, where it is focused 
onto a single-element detector by a focusing lens. The images on the DMD plane and detector are shown in Figure 2 (a) and 2 (b). As can be noticed, different wavelengths are dispersed on the DMD chip along the horizontal axis and channels are separated along the vertical axis.

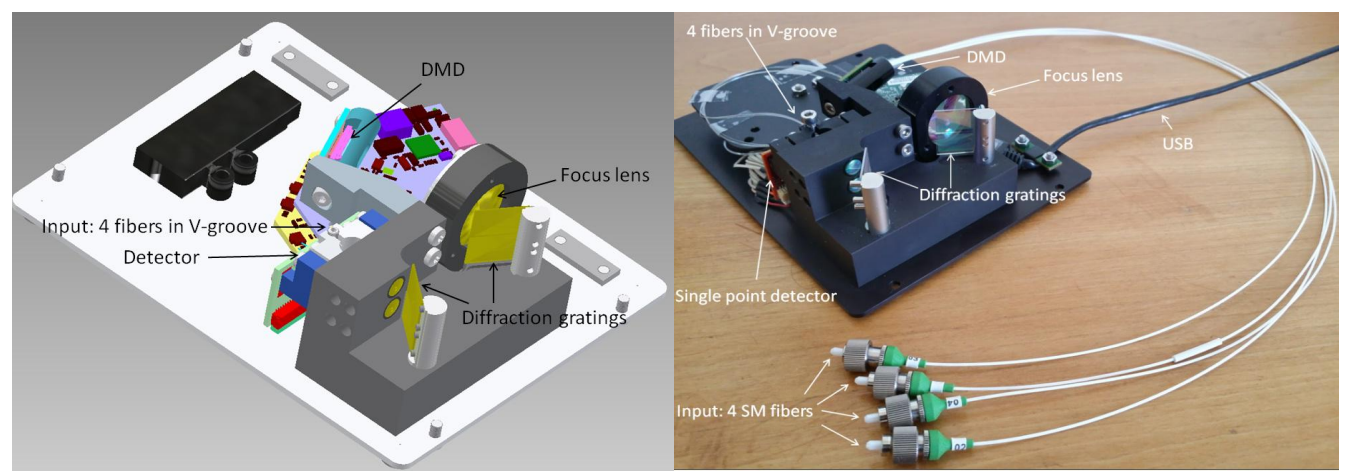

Figure 1. Spectrometer design: (a) left - schematic layout; (b) right - assembled prototype.

The design has been made in such a way to avoid channel cross talk and overlap. Here we use the 2D digital nature of the DMD chip and scan each channel one by one. The channels are also greatly separated in the detector plane, which potentially allows to replace one single-chip detector by a detector per channel to perform parallel channel readout. The unit has an USB interface to communicate with a PC.

(a)

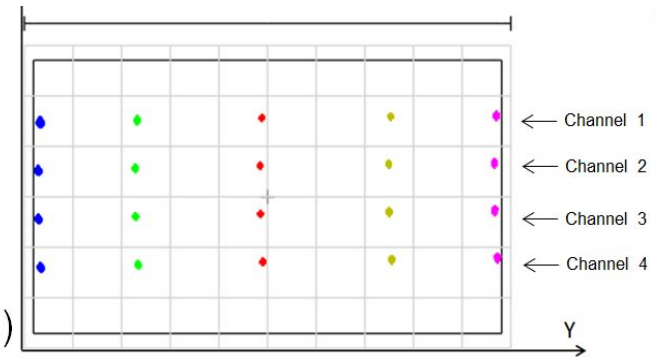

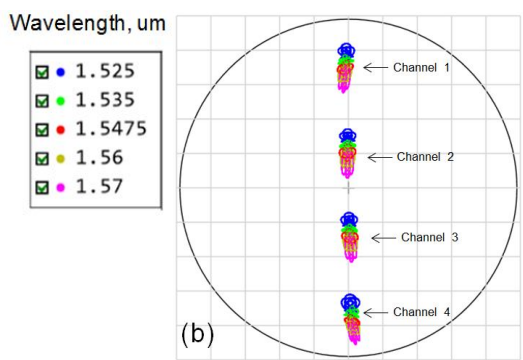

Figure 2. (a) Footprint of 5 wavelengths and 4 channels in the DMD plane; (b) footprint diagram on the detector, when all mirrors are in the $\mathrm{ON}$ state.

\section{PERFORMANCE}

\subsection{Optical resolution}

Figure 3 (a) illustrates measurements of the optical resolution, which is usually defined as Full Width at Half Maximum (FWHM). The FWHM varries from $120 \mathrm{pm}$ for Channel 1 to $165 \mathrm{pm}$ for Channel 4 . The presented values show that the optical resolution is good enough to clearly resolve even very sharp single-mode FBG peaks with $200 \mathrm{pm}$ width.

\subsection{Wavelength fit resolution}

The basic principle of FBG sensing is to track the FBG peak position. The resolution in the detected FBG peak position is often called Wavelength Fit Resolution (WFR) and mainly depends on 3 parameters [4]: a) signal-to-noise ratio (SNR) of the input signal; b) peak shape of the measured signal; c) selection of the fitting algorithm.

The most important parameter is the SNR of the measured signal. To investigate this dependence and exclude the other factors as input we used a tunable laser source with Gaussian shaped peak with fixed wavelength. The output power has been varied from -7 to $-65 \mathrm{dBm}$ with step of $1 \mathrm{~dB}$. The WFR was calculated as the standard deviation over 100 measurements per each value of the output laser power. The integration time was constant during the whole experiment. Figure 3 (b) shows the WFR vs. input power calculated for all channels. Despite slightly different optical resolution, the fit resolution is the same for all 4 channels. The dynamic range, where the WFR is less than $1 \mathrm{pm}$, is $39 \mathrm{~dB}$.

We have also carried out several experiments, where we test repeatability and thermal stability of the spectrometer. At the first experiment the polarization of the input light was rotated 360 degree. The repeatability is defined as the peak-topeak variation of the measured wavelength. The repeatability was measured to be less than $2 \mathrm{pm}$. On the second 
experiment the spectrometer was heated up to $50 \mathrm{C}$, then cooled down to $0 \mathrm{C}$ and then the temperature was set back to 25 C. The maximum wavelength shift is $5 \mathrm{pm}$ after the full cycle. These results show that the spectrometer is robust and well-suited for both industrial-grade applications and lab-type experiments.
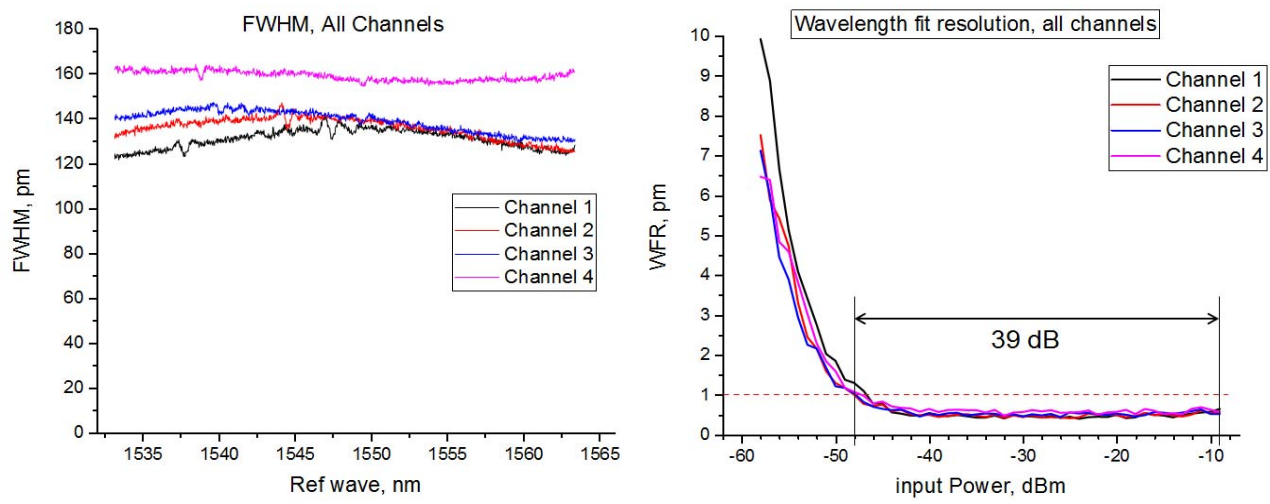

Figure 3. (a) left - measured FWHM; (b) right - wavelength fit resolution (WFR) vs. input power.

\subsection{Hadamard spectroscopy}

The simplest scheme of measuring a spectrum using the DMD is detecting one wavelength at the time by turning on micromirror columns one by one through the whole spectrum. Another scheme to acquire a spectrum is Hadamard spectroscopy [5]. The main advantage of this method is the improved SNR compared to the standard scheme. The digital nature of the DMD allows to implement this method. The idea of the Hadamard scan is to use special patterns, which can be generated from the Hadamard matrices. These patterns have always half of the mirrors in the on state, making the intensity of the detected signal higher and random noise lower compared to the standard sweep column scan. The SNR increases $\sqrt[3]{n} / 2$ times compared to sweep spectroscopy [5], where $\mathrm{n}$ is the number of mirrors used. The output spectrum is calculated by multiplying the measured values by the inverse Hadamard matrix. According to the theory, the SNR increases 12.2 times, which is $10.9 \mathrm{~dB}$, for $n=600$.

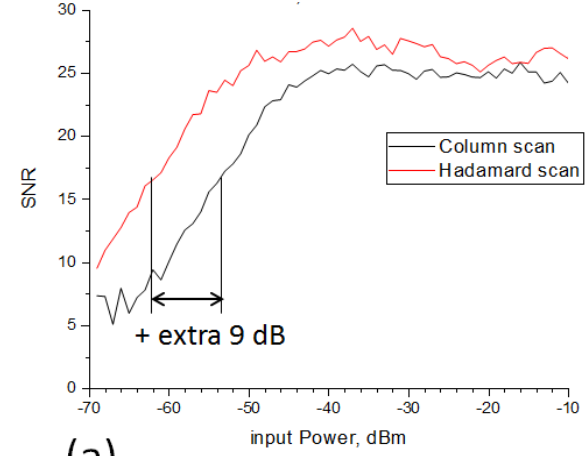

(a)

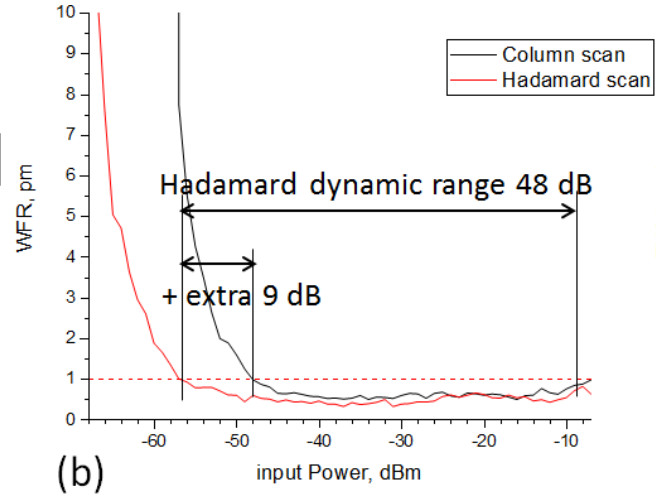

(b)

Figure 4. Comparison between the Hadamard and standard sweep scan methods: (a) SNR; (b) Wavelength fit resolution.

Figure 4 (a) shows an improvement of $9 \mathrm{~dB}$ in the SNR between the Hadamard and column scan methods, which is close to the theoretically predicted value. Here we used the same laser source as in the previous section. Figure 4 (b) confirms the fact that the WFR strongly depends on the SNR - the increase of the dynamic range is also $9 \mathrm{~dB}$. The dynamic range equals spectacular $48 \mathrm{~dB}$ for the Hadamard scan method.

Figure 5 shows a reflected spectrum of an FBG measured using the standard sweep column scan (a) and the Hadamard technique (c). As can be easily noticed, on the top left image the FBG peak is barely visible and the noise is very high. The WFR is around 16.7 pm (Fig. 5 (b)). When the Hadamard method was used to interrogate the same FBG, the WFR was improved up to 1.9 pm (Fig. 5 (d)) and the spectrum contains less noise and the FBG peak can be clearly distinguished (see Fig. 5 (c)). 

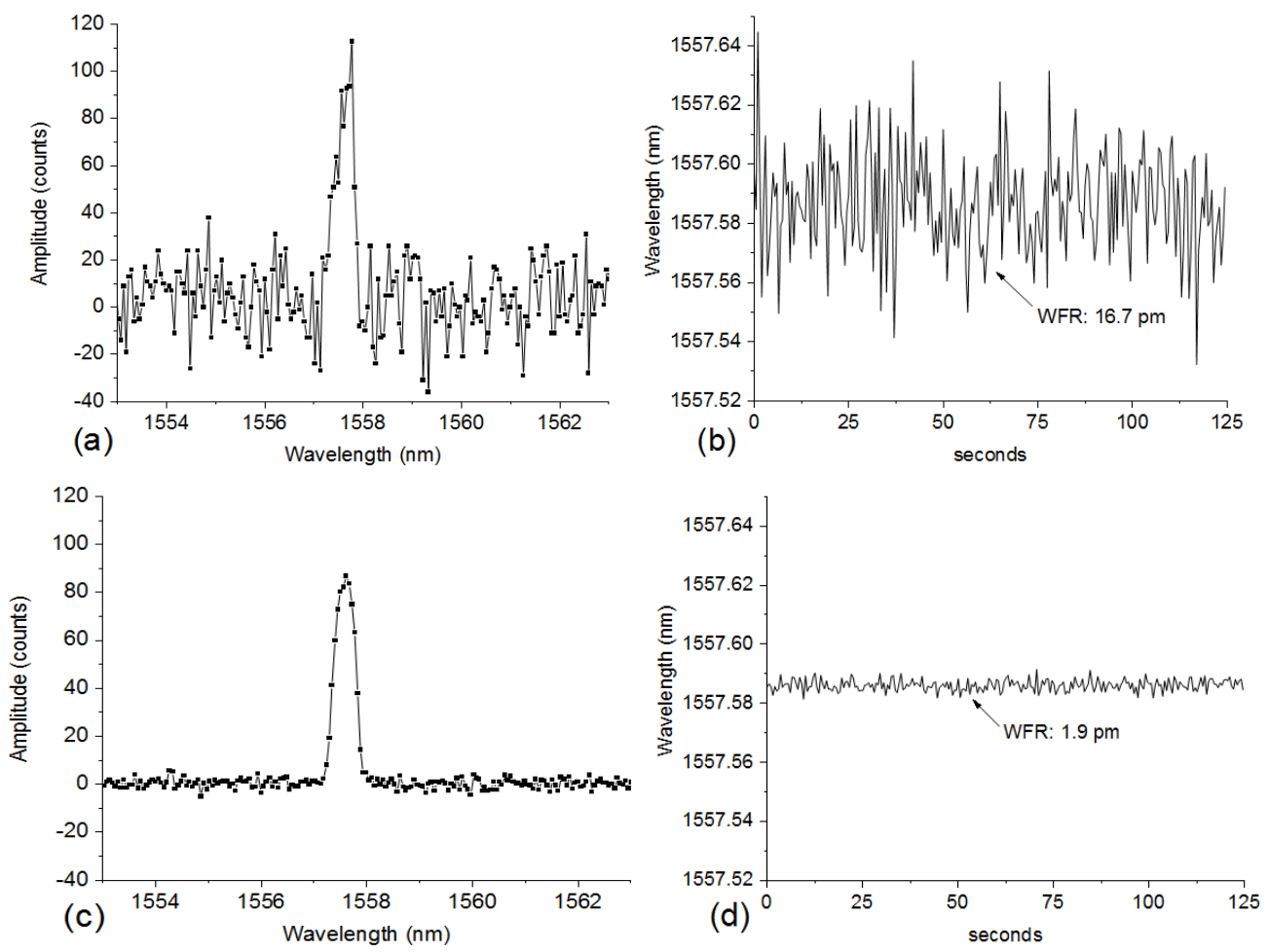

Figure 5. Column scan method: (a) FBG reflection spectrum; (b) tracked WL vs time; Hadamard technique: (c) FBG reflection spectrum; (d) tracked WL vs time.

\section{CONCLUSION}

In this paper we presented a novel type of multichannel MEMS based spectrometer, where the linear detector is replaced with a Digital Micromirror Device (DMD). The DMD is typically cheaper and has better pixel sampling than an InGaAs detector used in the $1550 \mathrm{~nm}$ range, which leads to cost reduction and better performance. Our measurements show that DMDs can replace standard InGaAs array detectors in high resolution spectroscopy and can be used in industrial-grade applications. Since the DMD is a 2D array, multichannel system has been implemented without any additional optical components in the spectrometer. In addition, the digital nature of the DMD provides opportunities for advanced programmable spectroscopic techniques, such a Hadamard spectroscopy, which greatly increase the SNR and wavelength fit resolution.

\section{ACKNOWLEDGMENTS}

The research leading to these results has received funding from the People Programme (Marie Curie Actions) of the European Union's Seventh Framework Programme FP7/2007-2013/ under REA grant agreement n 608382.

\section{REFERENCES}

[1] Kersey, A. D., Davis, M. A., Patrick H. J., LeBlanc, M., Koo, K. P., Askins, C. G., Putnam, M. A. and Friebele, E.J., "Fiber grating sensors," J. Lightwave Technol., 15, 1442-1442 (1997).

[2] Texas Instruments Data Sheet "DLP2010NIR 0.2 WVGA Near-Infrared DMD” (2015).

[3] Rose, B., Rasmussen, M., Herhold-Rasmussen, N., Jespersen, O., "Programmable spectroscopy enabled by DLP," Proc. of SPIE Vol. 9376 (2015).

[4] Ganziy, D., Jespersen, O., Woyessa, G., Rose, B. and Bang, O., "Dynamic gate algorithm for multimode fiber Bragg grating sensor system," Appl. Opt. 54, 5657-5661 (2015).

[5] Harwit, M. and Sloane, N. J. A., "Hadamard transform optics," Academic Press, USA, New York, 1979, chapter 2 . 